\title{
Upregulated $\beta$-catenin signaling does not affect survival of pancreatic cancer cells during dual inhibition of GSK3B and HDAC
}

\author{
Kateryna Nesteruk, Ron Smits, Marco Bruno, Maikel P. Peppelenbosch, Gwenny M. Fuhler* \\ Department of Gastroenterology and Hepatology, Erasmus University Medical Center Rotterdam, the Netherlands
}

\section{A R T I C L E I N F O}

\section{Article history:}

Received 21 August 2019

Received in revised form

10 December 2019

Accepted 23 December 2019

Available online $\mathrm{xxx}$

\section{Introduction}

The newly synthesized molecule Metavert was recently introduced as a promising new agent for treatment of pancreatic ductal adenocarcinoma (PDAC) [1]. Metavert slows tumor growth and metastasis by inhibiting both glycogen synthase kinase 3 beta (GSK3B) and histone deacetylases (HDACs). Edderkaoui et al. recently demonstrated that dual targeting of these pathways induces synergistic PDAC killing [1], and showed that Metavert decreases expression of cancer stemness markers associated with epithelial-to-mesenchymal transition and metastasis, which can still occur under inhibition of GSK3B alone [2]. However, an unexpected increase in $\beta$-catenin protein levels was seen in Metavert-treated PDAC cells, suggesting activation of $\mathrm{Wnt} / \beta$ catenin signaling. Wnt $/ \beta$-catenin signaling is complex and was shown to enhance PDAC development and malignancy [3-6]. However, it has also been suggested that $\beta$-catenin partly mediates killing effects of GSK3B inhibitors in KRAS-dependent tumors [7]. Furthermore, a specific dosage of $\beta$-catenin signaling is needed for tumor formation as an excessive accumulation of $\beta$ catenin leads to apoptosis in normal and carcinoma cells [8-10]. Thus, to what extent Wnt/ $\beta$-catenin signaling plays a role in Metavert-mediated PDAC killing remains unclear. We therefore investigated the activity of this pathway upon inhibition of GSK3 and/or HDAC and determined its role in PDAC cell cytotoxicity.

\section{Methods}

MTT test. BxPC-3, Panc-1, and MIAPaCa-2 cell lines were treated with GSK3B inhibitors CHIR99021 and TWS119, HDAC inhibitor Vorinostat or Wnt3a conditioned medium. MTT test was performed after $72 \mathrm{~h}[11]$.

$\beta$-Catenin Reporter Assays were performed as described [12]. After transfection with Wnt Responsive Element (WRE) or Mutant Responsive Element (MRE) vectors and TK-Renilla, luciferase activity was measured and normalized for transfection efficiency using the Dual Luciferase Reporter Assay system (Promega). WRE/ MRE ratios are shown.

$q P C R$ for Axin2 was performed as described [13]. In short, after $24 \mathrm{~h}$ of treatment total RNA was isolated for cDNA preparation. Primers used: forward TATCCAGTGATGCGCTGAC, reverse TTACTGCCCACACGATAAGG.

siRNA-mediated gene knock-down. Smartpool ON-TARGETplus siRNAs targeting CTNNB1 and nontargeting siRNA control \#2 were introduced into cells using DharmaFECT. Successful knockdown was confirmed by Western blot analysis [14] using a $\beta$-catenin antibody (\#610154, BD Transduction Laboratories) and IRdye-linked secondary antibodies. $\beta$-actin served as loading control.

Statistics. One-way ANOVA was used for statistical analysis of MTT, two-way ANOVA and student t-test was used for reporter assay and knockdown experiment. For dose-response curves, best fit sigmoidal dose-response (variable slope) curves are presented.

\footnotetext{
* Corresponding author.Department of Gastroenterology and Hepatology, Erasmus Medical Center, Wytemaweg 80, 3015 CNRotterdam, the Netherlands.

E-mail address: g.fuhler@erasmusmc.nl (G.M. Fuhler).
} 


\section{Results}

The $\beta$-catenin reporter assay showed that while the HDAC inhibitor Vorinostat alone does not change $\beta$-catenin signaling in PDAC cells (Fig. 1A, B, 1C), the GSK3B inhibitor CHIR99021 activates this pathway in a dose dependent fashion, far exceeding $\beta$ catenin signaling induced by Wnt-3a conditioned medium. Importantly, dual targeting of GSK3B and HDAC causes synergistic $\beta$-catenin activation as compared to CHIR99021 alone. Furthermore, in line with previous reports [15-17], the less potent GSK3B inhibitor TWS119 showed lower activation of $\beta$ catenin signaling (Fig. 1, Supplementary Fig. 1), but nevertheless also displayed synergistic $\beta$-catenin activation in Panc- 1 and BxPC-3 cells in combination with HDAC inhibition. Vorinostat also strengthens this signal in combination with Wnt-3a conditioned medium. We verified these findings by investigation of mRNA levels of AXIN2, a downstream target gene of $\beta$-catenin (Fig. 1D, E, 1F). Similar to CHIR99021, albeit at lower levels, TWS119 increases expression of AXIN2 alone and in combination with Vorinosat in all three cell lines.

We next investigated whether this synergistic $\beta$-catenin signaling mediates PDAC cell killing. First we confirmed the effect of combined inhibition of GSK3B and HDAC on PDAC growth inhibition (Fig. 2). Subsequently, we tested whether $\beta$-catenin
A

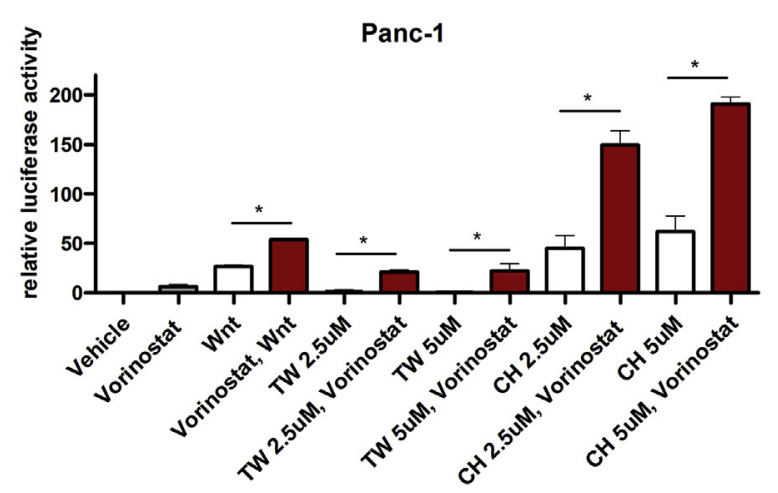

B

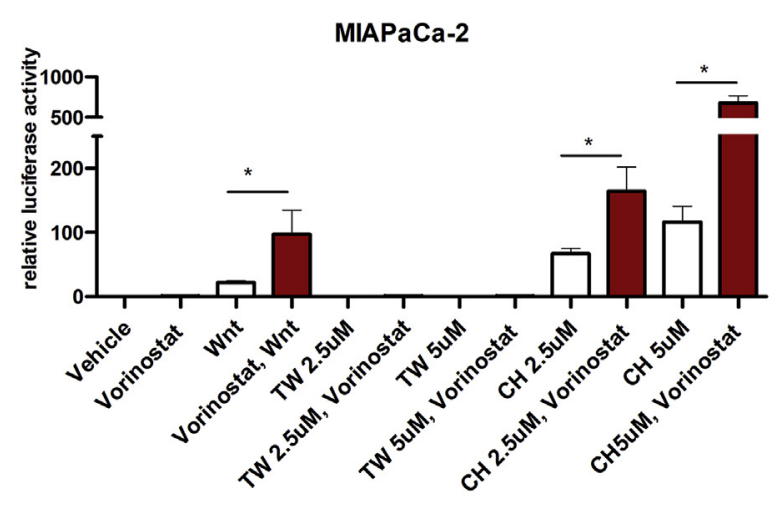

C

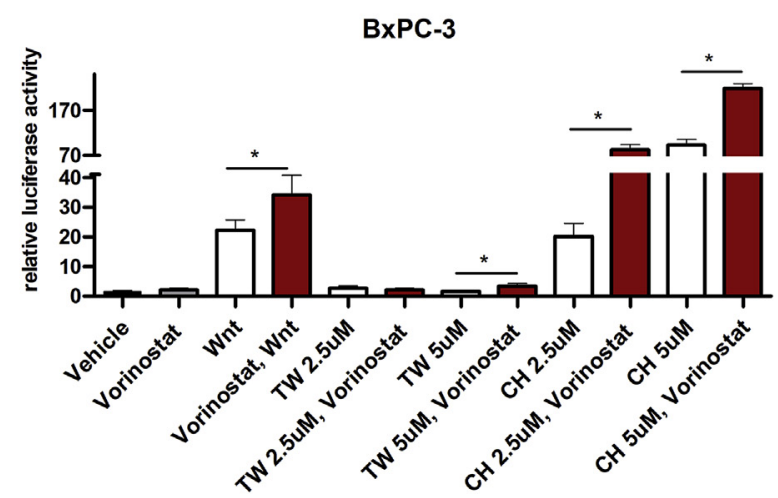

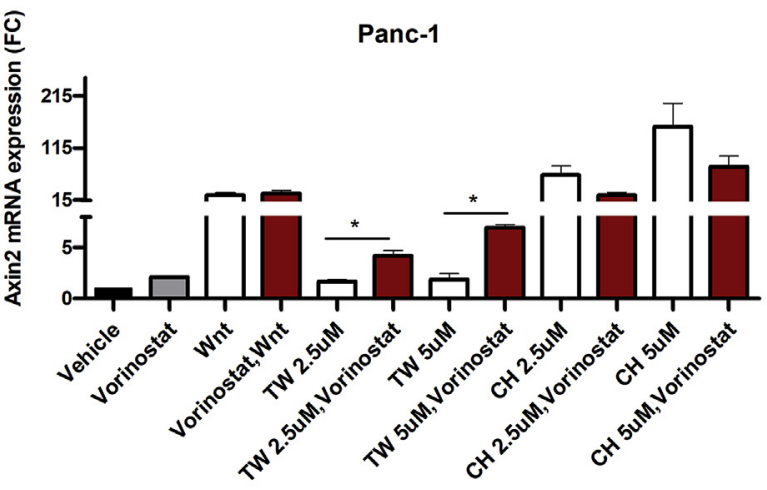

E

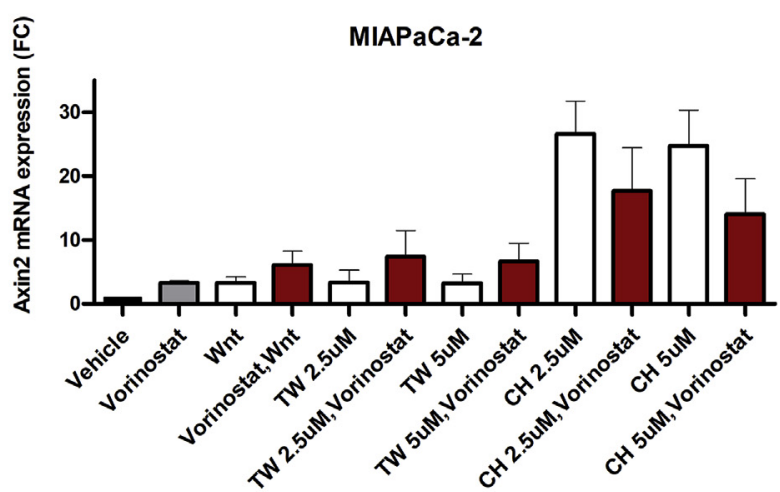

$\mathbf{F}$

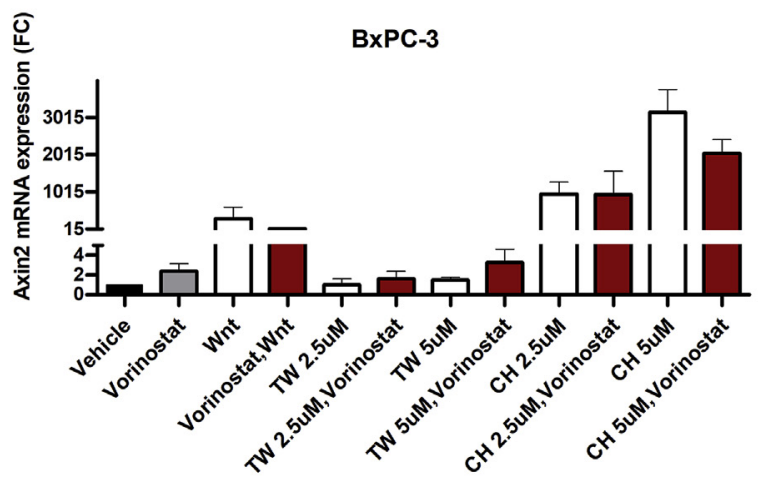

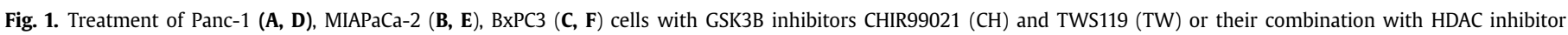
Vorinostat shows synergistic effect of these inhibitors on $\beta$-catenin signaling as determined by reporter assays or qPCR for AXIN2. Mean \pm SEM, ${ }^{*} \mathrm{p}<0.05$. 


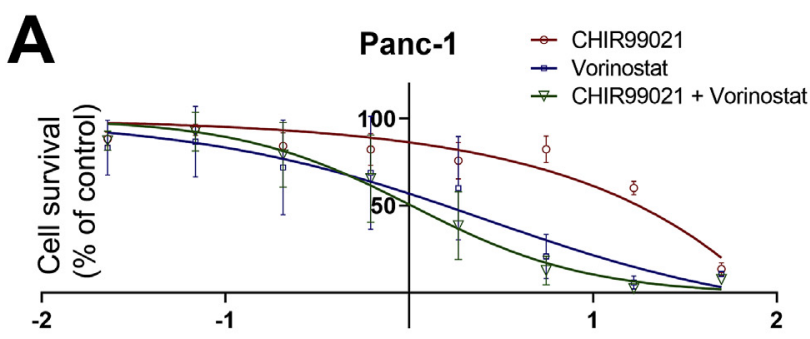

Conc. LOG, uM

B

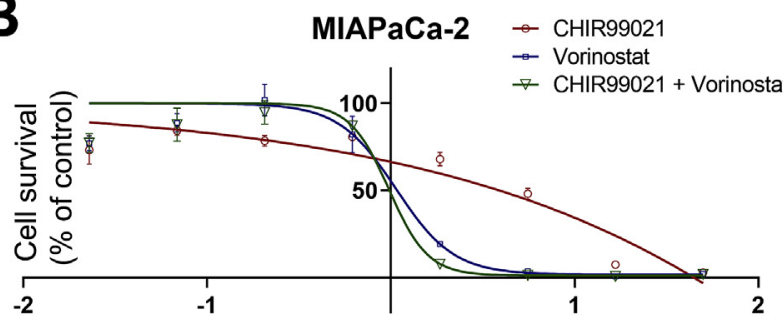

Conc. LOG, uM

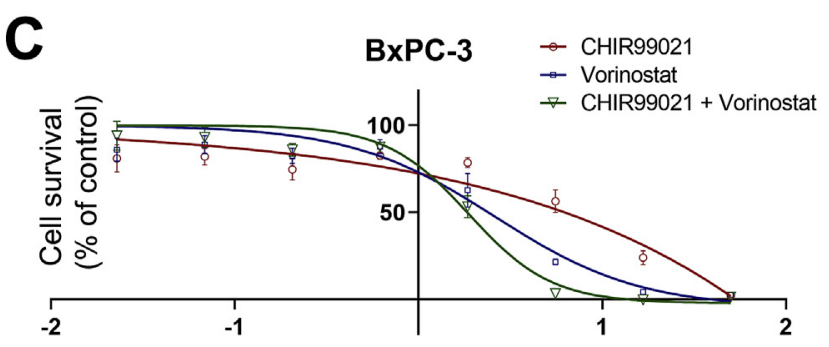

Conc. LOG, UM
D

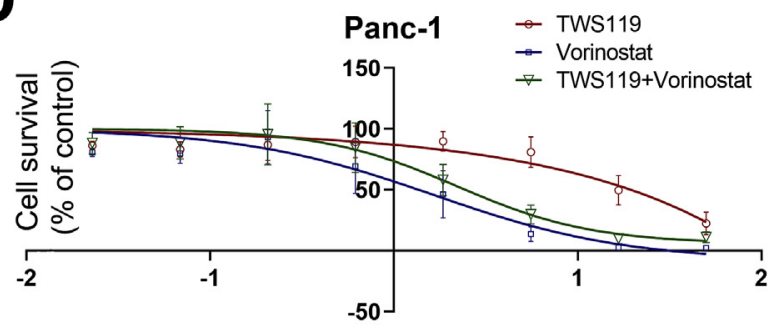

Conc. LOG, UM

$\mathbf{E}$

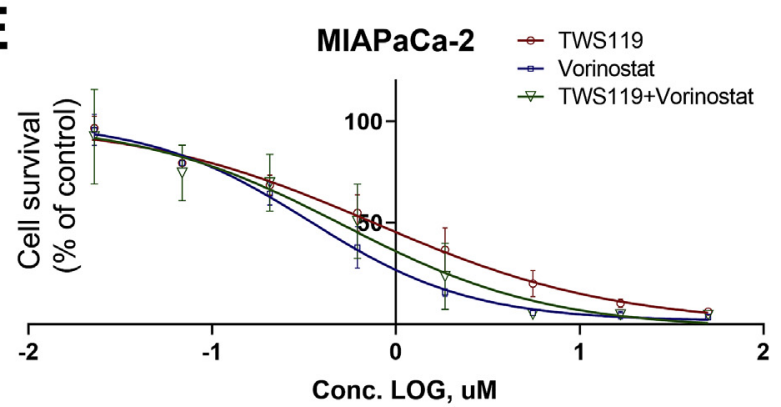

$\mathbf{F}$

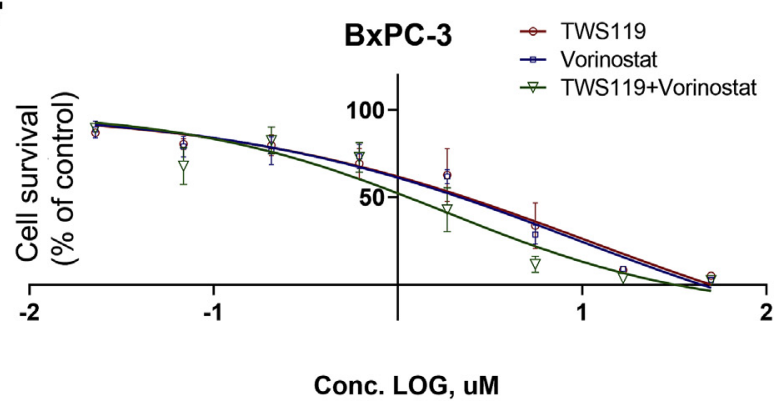

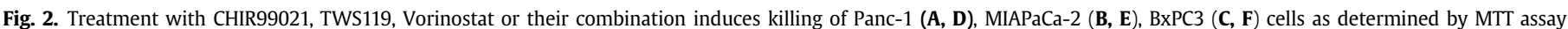
$($ Mean \pm SEM) $(B)$.

signaling affects PDAC growth by addition of Wnt-3a conditioned medium, as activation of Wnt3a receptors Frizzled and LRP5/6 leads to stabilization of cytoplasmic $\beta$-catenin [18]. However, Wnt3a stimulation did not affect PDAC cell viability (Fig. 3).

We then studied the direct effect of $\beta$-catenin through verified

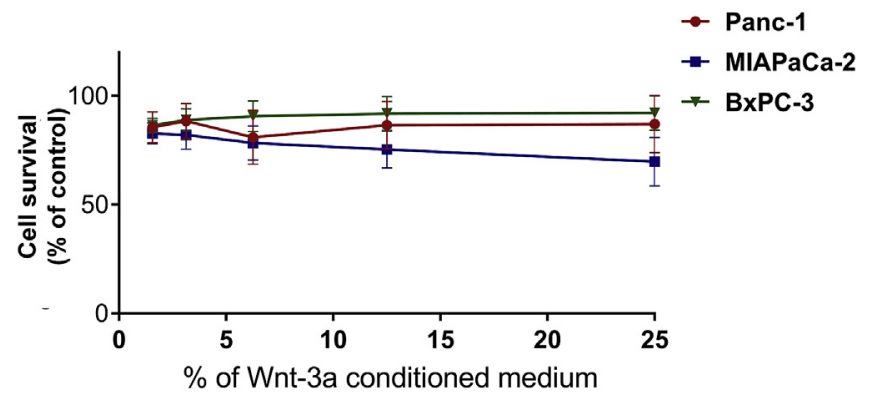

Fig. 3. Wnt-3a-conditioned medium does not affect cell viability as determined by MTT assay (Mean \pm SEM).
siRNA mediated knockdown (Fig. 4). Although knockdown of $\beta$ catenin in itself decreased the growth of pancreatic cancer cell lines by 22\% (Panc-1), 33\% (MIAPaCa-2) and 20\% (BxPC-3)(not shown, p < 0.05), knockdown did not affect susceptibility of Panc-1 and MIAPaCa-2 to treatment of GSK3B and HDAC inhibitor, and only slightly increased survival of BxPC-3 compared to controls when treated with CHIR99021 and Vorinostat (Fig. 4D).

\section{Discussion}

Our data suggest that while $\beta$-catenin knockdown in itself may reduce PDAC viability to some extent, activation of this signaling pathway does not contribute to the cytotoxic effects induced by combined GSK3/HDAC inhibition. It is conceivable that the synergistic $\beta$-catenin signaling triggered by these inhibitors seen here mediates other anti-tumorigenic effects of this dual treatment i.e. EMT or metastasis. The exact role of $\beta$-catenin signaling during Metavert treatment of PDAC remains elusive and requires further investigation. 
A

Panc-1 MIAPaCa-2 BxPC-3

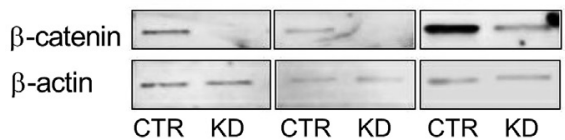

C

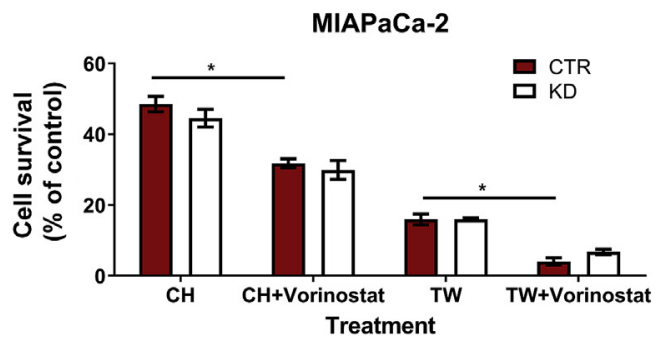

B

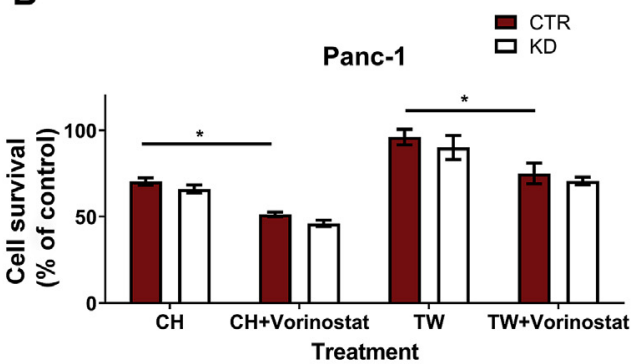

D

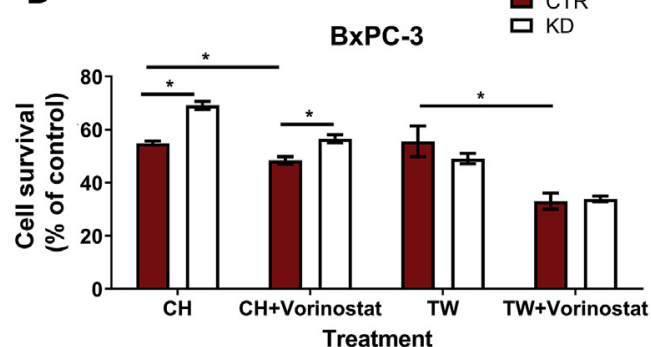

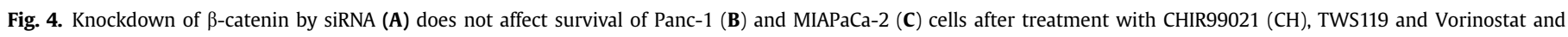
moderately inhibits cell killing of BxPC-3 (D) cells by $\mathrm{CH}$ and $\mathrm{CH}+$ Vorinostat (Mean $\pm \mathrm{SEM}$, *p $<0.05$ ).

\section{Author's contribution}

K. N.: acquisition of data, analysis and interpretation of data; statistical analysis; drafting of the manuscript.

R.S.: analysis and interpretation of data; study design; study supervision.

M. B: critical revision of the manuscript for important intellectual content; study supervision, technical, or material support

M.P.P: analysis and interpretation of data; critical revision of the manuscript for important intellectual content; technical, or material support

G.M.F: study concept and design, analysis and interpretation of data; drafting of the manuscript; critical revision of the manuscript for important intellectual content; study supervision.

\section{Declaration of competing interest}

All authors declare no conflict of interest regarding this study.

\section{Appendix A. Supplementary data}

Supplementary data to this article can be found online at https://doi.org/10.1016/j.pan.2019.12.019.

\section{References}

[1] Edderkaoui M, Chheda C, Soufi B, et al. An inhibitor of GSK3B and HDACs kills pancreatic cancer cells and slows pancreatic tumor growth and metastasis in mice. Gastroenterology 2018;155:1985-1998.e5.

[2] Zheng H, Li W, Wang Y, et al. Glycogen synthase kinase-3 beta regulates Snail and $\beta$-catenin expression during Fas-induced epithelial-mesenchymal transition in gastrointestinal cancer. Eur J Cancer 2013;49:2734-46.

[3] Sano M, Driscoll DR, DeJesus-Monge WE, et al. Activation of WNT/betaCatenin signaling enhances pancreatic cancer development and the malignant potential via up-regulation of Cyr61. Neoplasia 2016;18:785-94.

[4] Wang L, Heidt DG, Lee CJ, et al. Oncogenic function of ATDC in pancreatic cancer through Wnt pathway activation and beta-catenin stabilization. Cancer Cell 2009;15:207-19.

[5] Wang L, Yang H, Abel EV, et al. ATDC induces an invasive switch in KRASinduced pancreatic tumorigenesis. Genes Dev 2015;29:171-83.

[6] Yu M, Ting DT, Stott SL, et al. RNA sequencing of pancreatic circulating tumour cells implicates WNT signalling in metastasis. Nature 2012;487:510-3.

[7] Kazi A, Xiang S, Yang H, et al. GSK3 suppression upregulates $\beta$-catenin and cMyc to abrogate KRas-dependent tumors. Nat Commun 2018;9:5154.

[8] Albuquerque C, Breukel C, van der Luijt R, et al. The 'just-right' signaling model: APC somatic mutations are selected based on a specific level of activation of the $\beta$-catenin signaling cascade. Hum Mol Genet 2002;11:1549-60.

[9] Kim K, Pang KM, Evans M, et al. Overexpression of beta-catenin induces apoptosis independent of its transactivation function with LEF-1 or the involvement of major G1 cell cycle regulators. Mol Biol Cell 2000;11:3509-23.

[10] Albuquerque C, Bakker ERM, van Veelen W, et al. Colorectal cancers choosing sides. Biochim Biophys Acta Rev Canc 2011;1816:219-31.

[11] Queiroz KC, Milani R, Ruela-de-Sousa RR, et al. Violacein induces death of resistant leukaemia cells via kinome reprogramming, endoplasmic reticulum stress and Golgi apparatus collapse. PLoS One 2012;7:e45362.

[12] Bakker ER, Das AM, Helvensteijn W, et al. Wnt5a promotes human colon cancer cell migration and invasion but does not augment intestinal tumorigenesis in Apc1638N mice. Carcinogenesis 2013;34:2629-38.

[13] Janmaat VT, Liu H, da Silva RA, et al. HOXA9 mediates and marks premalignant compartment size expansion in colonic adenomas. Carcinogenesis 2019 Feb 20:1-11. https://doi.org/10.1093/carcin/bgz038. pii: bgz038.

[14] Somasundaram R, Fernandes S, Deuring JJ, et al. Analysis of SHIP1 expression and activity in Crohn's disease patients. PLoS One 2017;12. e0182308.

[15] Billin AN, Thirlwell H, Ayer DE. Beta-catenin-histone deacetylase interactions regulate the transition of LEF1 from a transcriptional repressor to an activator. Mol Cell Biol 2000;20:6882-90.

[16] Bordonaro M, Lazarova DL, Sartorelli AC. The activation of beta-catenin by Wnt signaling mediates the effects of histone deacetylase inhibitors. Exp Cell Res 2007;313:1652-66.

[17] Debeb BG, Lacerda L, Xu W, et al. Histone deacetylase inhibitors stimulate dedifferentiation of human breast cancer cells through WNT/ $\beta$-catenin signaling. Stem cells (Dayton, Ohio) 2012;30:2366-77.

[18] MacDonald BT, He X. Frizzled and LRP5/6 receptors for Wnt/ $\beta$-catenin signaling. Cold Spring Harbor perspectives in biology;4:a007880. 